\title{
Elliptical optical tweezers for trapping a red blood cell aggregate
}

\author{
Antti J. Kauppila, ${ }^{1}$ Matti T. Kinnunen, ${ }^{* 1}$ Artashes Karmenyan ${ }^{2}$, and Risto A. Myllylä ${ }^{1}$ \\ ${ }^{1}$ Department of Electrical and Information Engineering, Technical Faculty, University of Oulu, Oulu, Finland \\ ${ }^{2}$ Institute of Biophotonics, National Yang Ming University, Taipei, Taiwan
}

Received May 18, 2011; accepted July 16, 2011; published September 30, 2011

\begin{abstract}
Red blood cells (RBCs) and their aggregates scatter light strongly. Optical tweezers facilitate the manipulation of cells and light scattering measurements at the single cell level. We show in this letter the successful trapping of an RBC aggregate with elliptical optical tweezers in a free suspension. Two cylindrical lenses are used to form the trap. The distance between the trapped objects and the bottom of the cuvette had a clear effect on image quality. Rotating the cylindrical lenses changes the orientation of the aggregate, which produces a visible change in the light scattering intensity images taken by a CCD camera.
\end{abstract}

The light scattering properties of biological tissues affect a sample's photon distribution during laser illumination. Apart from inducing blurred deep images of subcutaneous structures and decreasing light penetration depth, this will also have an adverse effect on the non-invasive measurement of blood constituents. Red blood cells (RBCs) are the main blood component, and they induce both strong absorption and strong scattering. Scattering properties are dependent on the refractive index of the scatterers as well as that of the base medium (extracellular medium or blood plasma) which affect blood osmolarity [1]. Scattering and light propagation are also affected by the size and shape of RBCs and their aggregation properties [1]-[5]. Optical clearing is a promising technique to decrease light scattering in a biological tissue [6]. The effects of different optical clearing agents (OCAs) on the optical properties of blood have been studied carefully [4]-[6]. Some clearing agents, such as various dextrans, induce RBC aggregation when added to blood plasma. However, optimal concentrations of OCAs serve to reduce the scattering properties of blood [7], which have been studied extensively both experimentally [8]-[10] and theoretically [11], [12]. In addition, several groups are investigating the optical properties of whole blood [1], [13], [14] as well as RBC aggregates at the whole blood level [3]-[5] and in RBC suspensions [15]. This helps the analysis and understanding of optical measurement results. Although theoretical information is available concerning light-matter interaction at the single RBC aggregate level [16], tools for single-cell studies are still under development. Some papers describe the optical trapping and manipulation of RBC aggregates using two

\footnotetext{
*E-mail: matti.kinnunen@ee.oulu.fi
}

point optical tweezers to study the RBC (dis)aggregation mechanism and to measure the present aggregation forces [17], [18]. Also laser interference patterns have been used to study the orientation and disaggregation of RCBs [19]. In this paper, we show the first successful trapping of an RBC aggregate with elliptical optical tweezers (to the authors' knowledge) with a water immersion objective. We then demonstrate the rotation of the aggregate's orientation and show changes in the near-field light scattering distribution of CCD camera images, when the aggregate is illuminated by $\mathrm{He}-\mathrm{Ne}$ laser light.

The optical trapping setup consists of a laser (ILML3IF-300 diode, Leadlight technology, Taiwan), two cubic beam splitters for dividing the laser beam into two separate beams to form the point and elliptical tweezers of the setup, as well as a microscope objective (Olympus LUMPlanFl 100x/1.00 $\mathrm{W} \infty / 0$ ) for making the traps. The elliptical optical tweezers are formed using two cylindrical lenses with a focal length of $75 \mathrm{~mm}$ and $150 \mathrm{~mm}$. All output power of the laser $(\mathrm{P} \sim 350 \mathrm{~mW})$ was directed into the elliptical beam. The sample was illuminated with white LEDs and the trapped cells were imaged by a CCD camera (LCL-902K Watec America Corp.). In a simplified form, Fig. 1 shows the shaping of a Gaussian beam into an elliptical beam by two cylindrical lenses. As seen, the trapped aggregates are located in a free suspension in a cylindrical cuvette (height $15 \mathrm{~mm}$, inner diameter $22.6 \mathrm{~mm}$ and outer diameter $25 \mathrm{~mm}$ (shortened version of Hellma 540.115)). A more detailed description of the setup can be found in Ref. [20], while Ref. [21] describes the He-Ne laser illumination part employed in the scattering measurements.

Fresh red blood cells were collected using the finger prick method and diluted with filtered PBS (phosphate buffered saline, D8537, Sigma, Sigma-Aldrich). This PBS was filtered three times with a filter pore size of $0.2 \mu \mathrm{m}$ (GELMAN Acrodisc 13 CR PTFE). For aggregate studies, PBS-diluted RBCs were added to a solution containing Dextran T500 (Pharmacia Fine Chemicals AB, Uppsala, Sweden) (D), bovine albumin (A-8022, Sigma Chemicals CO., USA) (A) and PBS (ratio: $3 \mathrm{ml} 3 x$ filtered PBS, 15mg Dextran T 500 and 30mg Albumin). Before adding the RBCs, the solution was filtered twice using the 
$0.2 \mu \mathrm{m}$ filter. A small portion of the aggregated RBCs were then added to the D+A+PBS-solution on the surface of the measurement cuvette.

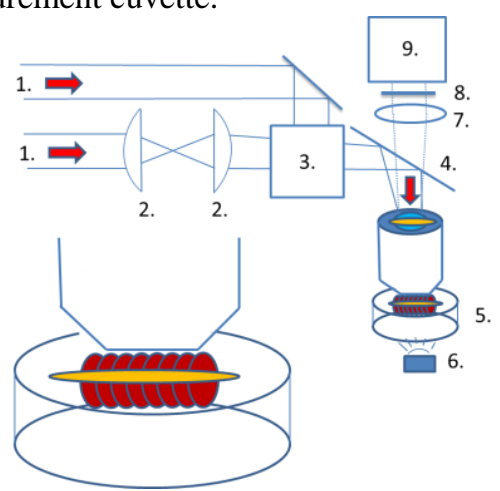

Fig. 1. Shaping of a Gaussian laser beam into an elliptical beam using two cylindrical lenses. 1. Collimated laser beam, 2 . cylindrical lenses, 3 . beam splitter, 4. dichroic mirror, 5. sample cuvette, 6. light for sample

illumination, 7. focusing lens, 8. IR filter, and 9. CCD camera.

In the first experiment, the optical tweezers were lifted well above the cuvette bottom. Free-flowing aggregated RBCs were then allowed to be trapped from the sample medium. Once an RBC aggregate was trapped, the rest of the RBCs were allowed to sedimentate to the bottom. Figure 2 illustrates the RBC aggregate in the elliptical optical tweezers. The aggregate was held in the trap for three and a half minutes at a distance of $750 \mu \mathrm{m}$ from the cuvette bottom. Distances to the side walls of the cuvette were $\sim 11.3 \mathrm{~mm}$. The optical power in the trap was $70.5 \pm 0.5 \mathrm{~mW}$.

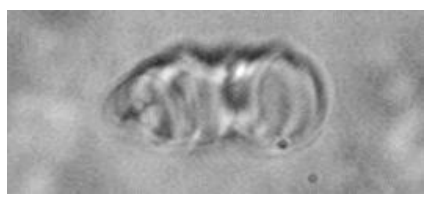

Fig. 2. Red blood cell aggregate in elliptical optical tweezers.

Being long, the aggregate was quite heavy and, as Fig. 2 shows, it sagged a bit in the middle. The sample was lit from the bottom of the cuvette, and as light propagated through the bottom and the sample, its intensity was attenuated by the sedimentated cells (and aggregates) and the slightly turbid base medium. Since the trapped aggregate was $750 \mu \mathrm{m}$ from the bottom, this attenuation led to a decrease in image quality. Also the long working distance of our objective $(1.5 \mathrm{~mm})$ affected image quality, because light also needed to pass this distance through the medium. As a result, the quality of the aggregate image in Fig. 2 is not as sharp as in some other papers [18] employing a thin sample cuvette and an objective with a much shorter working distance. While two-point tweezers allow forming and pulling down aggregates and measuring forces between cells [17], [18], they cannot be used to move and lift long aggregates.
We then evaluated the trapping efficiency of our elliptical tweezers. The $\mathrm{Q}$ value was measured and calculated using the viscous drag force method [22], taking account of changes in cell shape and the distance to the cuvette bottom [22]-[24]. It was found that, in trapping a single cell, the efficiency of elliptical tweezers is lower $(0.024 \pm 0.009)$ than that of point tweezers $(0.11 \pm 0.08)$, due to the different intensity distribution in the trap. However, several cells can be trapped at the same time [20], and the size of the elliptical tweezers is appropriate for trapping an RBC aggregate.

Two cylindrical lenses were used to produce an axial trapping force. This force was sufficiently strong to keep the trapped particles in the free suspension [20] at the necessary altitude from the bottom for the scattering measurements, in which the He-Ne laser was employed from the lateral face. Rotation of the RBC aggregate was accomplished by rotating the cylindrical lenses (2. in Fig. 1) to change the angle of the focused light line in the focal plane of the lens. Figure 3 shows six positions of erythrocyte rouleaux as they are gradually rotated by 10 degrees at each step. These images were taken at 1 to 4 minutes intervals. In this experiment, the aggregates were formed spontaneously and the number of cells was not controlled.

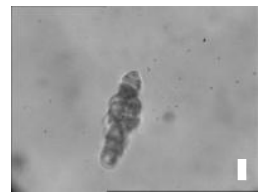

(a)

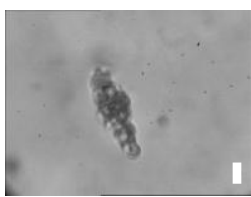

(d)

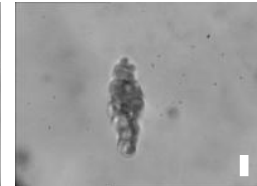

(b)

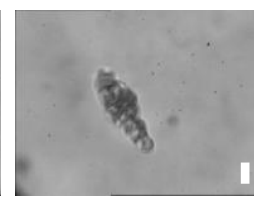

(e)

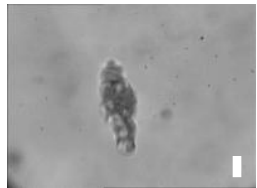

(c)

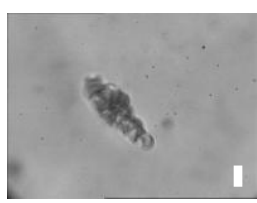

(f)
Fig. 3.Time-lapse image of rotating an RBC aggregate in elliptical optical tweezers. (scale bar is $10 \mu \mathrm{m}$ )

Trapping aggregates in a free suspension provides good conditions to study their light scattering properties. Elastic light scattering has been measured from single cells with various techniques. Some groups have used the goniometric method for measuring the scattering intensity of a cell under laser illumination [21], [25], [26]. For RBCs, however, most of the published results have been obtained through an image analysis of intensity images [27], [28]. Our recent results show the potential of the goniometric method for single RBC level measurements [29]. In these experiments, RBCs were suspended in a filtered PBS suspension. Filtered PBS is fully transparent for laser light and all microscale impurities have been 
removed, which is one of the most important criteria for a sample, when measuring elastic light scattering from single particles. A dextran-albumin-PBS solution does not satisfy this requirement, because some substances still scatter light and induce intensity fluctuations in the background signal.

The He-Ne laser was aligned at the same height from the bottom as the trapped aggregate. Figure 4 shows the first images of a single RBC aggregate under laser illumination. It is evident that the orientation of the aggregate affects the near-field intensity distribution. As seen, the scattering intensity is much stronger than from single cells [29], due to the aggregate's larger scattering cross-section.

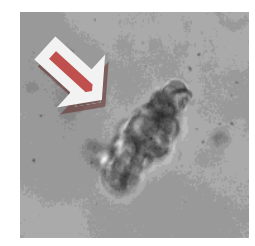

(a)

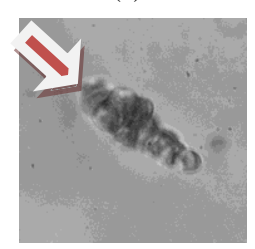

(c)

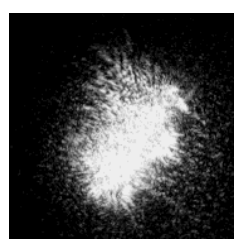

(b)

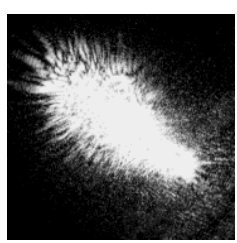

(d)
Fig. 4. Near-field light scattering intensity image from an RBC aggregate at two different orientations. The arrows show the direction of incident laser light.

By measuring the suspension's total refractive index and relative refractive index $\left(m=n_{p} / n_{t}\right.$, where $n_{p}$ is the refractive index of the particle and $n_{t}$ is the total refractive index of the medium), we can estimate the effect of an additive on light scattering from a single particle. When using a D+A+PBS solution (as described above) instead of PBS, $n_{t}$ increases by $1 \%$ and $m$ decreases by $0.7 \%$. In these calculations, we used the value of 1.40 for the $n_{p}$ of red blood cells [30], while the $n_{t}$ of the suspension was measured with optical coherence tomography. In whole blood, as in autologous plasma suspensions, plasma and different proteins induce RBC aggregation. By introducing a more clear suspension for aggregates, we might be able to improve the conditions for elastic light scattering measurements and enhance the quality of the CCD camera image of trapped aggregates.

As a conclusion, we have demonstrated that RBC aggregates can be trapped with elliptical optical tweezers. Moreover, the trapping efficiency is sufficient to lift and rotate the aggregates. We also demonstrated the first results of light-matter interaction at the single RBC aggregate level in a free suspension.

This work belongs to a project that has been funded by the Academy of Finland (124176). Dr. Kinnunen is thankful to the Academy of Finland for his personal grant (128073).

\section{References}

[1] A. Roggan, M. Friebel, K. Dörschel, A. Hahn, G. Müller, J. Biomed. Opt. 4, 36 (1999).

[2] A.N. Shvalov, J.T. Soini, A.V. Chernyshev, P.A. Tarasov, E. Soini, V.P. Maltsev, Appl. Opt. 38, 230 (1999).

[3] A.V. Priezzhev, O.M. Ryaboshapka, N.N. Firsov, I.V. Sirko, J. Biomed. Opt. 4, 76 (1999)

[4] V.V. Tuchin, X. Xu, R.K. Wang, Appl. Opt. 41, 258 (2002).

[5] X. Xu, R.K. Wang, J.B. Elder, V.V. Tuchin, Phys. Med. Biol. 48, 1205 (2003).

[6] V.V. Tuchin, Laser Phys. 15, 1109 (2005).

[7] A.N. Bashkatov, D.M. Zhestkov, É.A. Genina, V.V. Tuchin, Opt. Spect. 98, 638 (2005).

[8] K.A. Sem'yanov, P.A. Tarasov, J.T. Soini, A.K. Petrov, V.P. Maltsev, Appl. Opt. 39, 5884 (2000).

[9] M.A. Yurkin, K.A. Semyanov, P.A. Tarasov, A.V. Chernyshev, A.G. Hoekstra, V.P. Maltsev, Appl. Opt. 44, 5249 (2005).

[10] D.H. Tycko, M.H. Metz, E.A. Epstein, A. Grinbaum, Appl. Opt. 24, 1355 (1985).

[11] S.V. Tsinopoulos, D. Polyzos, Appl. Opt. 38, 5499 (1999).

[12] A.M.K. Nilsson, P. Alsholm, A. Karlsson, S. Andersson-Engels, Appl. Opt. 37, 2735 (1998).

[13] M. Friebel, J. Helfmann, M.C. Meinke, J. Biomed. Opt. 15, 055005 (2010).

[14] A.K. Amerov, J. Chen, G.W. Small, M.A. Arnold, Anal. Chem. 77, 4587 (2005).

[15] C.V.L. Pop, S. Neamtu, J. Biomed. Opt. 13, 041308 (2008).

[16] S.V. Tsinopoulos, E.J. Sellountos, D. Polyzos, Appl. Opt. 41, 1408 (2002).

[17] P.J.H. Bronkhorst, J. Grimbergen, G.J. Brakenhoff, R.M. Heethaar, J.J. Sixma, British J. Haematol. 96, 256 (1997).

[18] M.D. Khokhlova et al., Proc. SPIE 7715, 77150M (2010).

[19] J.V. Kruchenok, S.B. Bushuk, G.I. Kurilo, N.A. Nemkovich, A.N. Rubinov, J. Biol. Phys. 31, 73 (2005).

[20] A. Kauppila, M. Kinnunen, A. Karmenyan, R. Myllylä, manuscript under preparation.

[21] M. Collins, A. Kauppila, A. Karmenyan, L. Gajewski, K. Szewczyk, M. Kinnunen, R. Myllylä, Proc. SPIE 7376, 737619 (2010).

[22] W.H. Wright, G.J. Sonek, M.W. Berns, App. Opt. 33, 1735 (1994).

[23] D. Leith, Aerosol Sci. Technol. 6, 153 (1987).

[24] E. Loth, Powder Techol. 182, 342 (2008).

[25] R.M.P. Doornbos, M. Schaeffer, A.G. Hoekstra, P.M A. Sloot, B.G. de Grooth, J. Greve, Appl. Opt. 35, 729 (1996).

[26] D. Watson, N. Hagen, J. Diver, P. Marchand, M. Chachisvilis, Biophys. J. 87, 1298 (2004).

[27] W.H. Wright, G.J. Sonek, Y. Numajiri, M.W. Berns, Proc. SPIE 1427, 279 (1991).

[28] Y. Park, M. Diez-Silva, D. Fu, G. Popescu, W. Choi, I. Barman, S. Suresh, M.S. Feld, J. Biomed. Opt. Lett. 15, 020506 (2010).

[29] M. Kinnunen, A. Kauppila, A. Karmenyan, R. Myllylä, Biomed. Opt. Express 2, 1803 (2011).

[30] G.J. Streekstra, A.G. Hoekstra, E.-J. Nijhof, R.M. Heethaar, Appl. Opt. 32, 2266 (1993). 